\title{
The Latest on Apertif
}

\section{Tom Oosterloo*, Wim van Cappellen}

Netherlands Institute for Radio Astronomy - ASTRON

Postbus 2

7990 AA Dwingeloo

The Netherlands

E-mail: oosterloo@astron.nl

\section{Marc Verheijen}

Kapteyn Astronomical Institute

University of Groningen

Postbus 800

9700 AV Groningen

The Netherlands

\section{Laurens Bakker, George Heald, Marianna Ivashina}

Netherlands Institute for Radio Astronomy - ASTRON

Postbus 2

7990 AA Dwingeloo

The Netherlands

We describe a focal plane array (FPA) system, called Apertif, that is being developed for the Westerbork Synthesis Radio Telescope (WSRT). The aim of Apertif is, at frequencies from 1.0 to $1.7 \mathrm{GHz}$, to increase its instantaneous field of view by a factor of 37 and its observing bandwidth to $300 \mathrm{MHz}$ with high spectral resolution. This system will turn the WSRT into an effective survey telescope with scientific applications ranging from deep surveys of the northern sky of HI and $\mathrm{OH}$ emission and polarised continuum, to efficient searches for pulsars and transients. We present results obtained with a prototype FPA installed in one of the WSRT dishes. The results obtained with this prototype demonstrate that FPAs do have the required performance and that they can be used to turn radio telescopes into very effective survey instruments.

Panoramic Radio Astronomy: Wide-field 1-2 GHz research on galaxy evolution - PRA2009

June 02 - 052009

Groningen, the Netherlands

\footnotetext{
*Speaker.
} 


\section{Introduction}

Radio astronomy is a very successful branch of astronomy that has made several fundamental contributions to science, for example, the detection of the cosmic microwave background, the discovery of pulsars and detailed spectroscopic studies of the interstellar medium in the Milky Way and other galaxies. However, in order to keep the momentum of discovery, the performance of radio instruments has to be improved continuously. One of the main limitations of current radio telescopes is that, because of their limited field of view, it is very expensive in terms of observing time to observe large volumes of space with high sensitivity in order to detect sufficiently large samples of faint sources. Such deep, wide-field studies are called for in the context of many very relevant astronomical topics, such as the evolution of galaxies and the properties of the transient sky. The obvious solution to this problem is to replace the single-feed systems currently employed in many radio telescopes with arrays of detectors. This effectively turns a radio dish into a radio camera. On single dish radio telescopes this has been done by installing multiple feeds in the focal plane which provide several beams on the sky, such as the successful Parkes multi-beam system which surveyed the entire southern sky in HI [6],[5], and the Arecibo multi-beam system AlfAlfa [3].

Current technology offers a method to form multiple beams on the sky in a more efficient way. The basic idea is to employ phased array technology in the focal plane of a radio telescope. The advantage of this technique is, in contrast to the multi-horn systems mentioned above, that the radiation field in the focal plane is fully sampled. This offers much greater flexibility in forming many beams on the sky and allows, at the same time, to optimise the performance of the telescope dish for maximum gain or other properties. Apertif ("APERture Tile In Focus") is such a system which is being developed for the Westerbork Synthesis Radio Telescope (WSRT). The aim of Apertif is to turn the WSRT into an instrument that will perform deep all-northern-sky imaging surveys in spectral line and polarised continuum. In this paper we will briefly describe the technical details of this focal plane array (FPA) system under development and report first results with a prototype FPA installed in one of the WSRT dishes. A discussion of possible HI surveys with Apertif is given by [7].

\section{The APERTIF system and its prototype}

The development of the Apertif FPA system builds upon many years of experience with phased arrays at ASTRON, an effort motivated by enabling this technology for the Square Kilometre Array [4],[1]). The design specifications for the APERTIF FPA system are that it should operate in the frequency range of $1000-1750 \mathrm{MHz}$, using an instantaneous bandwidth of $300 \mathrm{MHz}$ covered with 16384 channels, a system temperature of $50-55 \mathrm{~K}$ and an aperture efficiency of $75 \%$. For the WSRT this amounts to an effective area to system temperature ratio of $A_{\text {eff }} / T_{\text {sys }} \sim 100 \mathrm{~m}^{2} / \mathrm{K}$, if all 14 telescopes are equipped with FPAs. The goal is to have 37 beams on the sky simultaneously, giving an effective field of view (FoV) of 8 square degrees. This entire field of view will be imaged with 15 arcseconds resolution over a bandwidth of $300 \mathrm{MHz}$ with a spectral resolution of about $4 \mathrm{~km} \mathrm{~s}^{-1}$. The survey speed of Apertif, and many of the other characteristics, will be very similar to ASKAP, an FPA-equipped telescope which is being constructed in Australia by the 


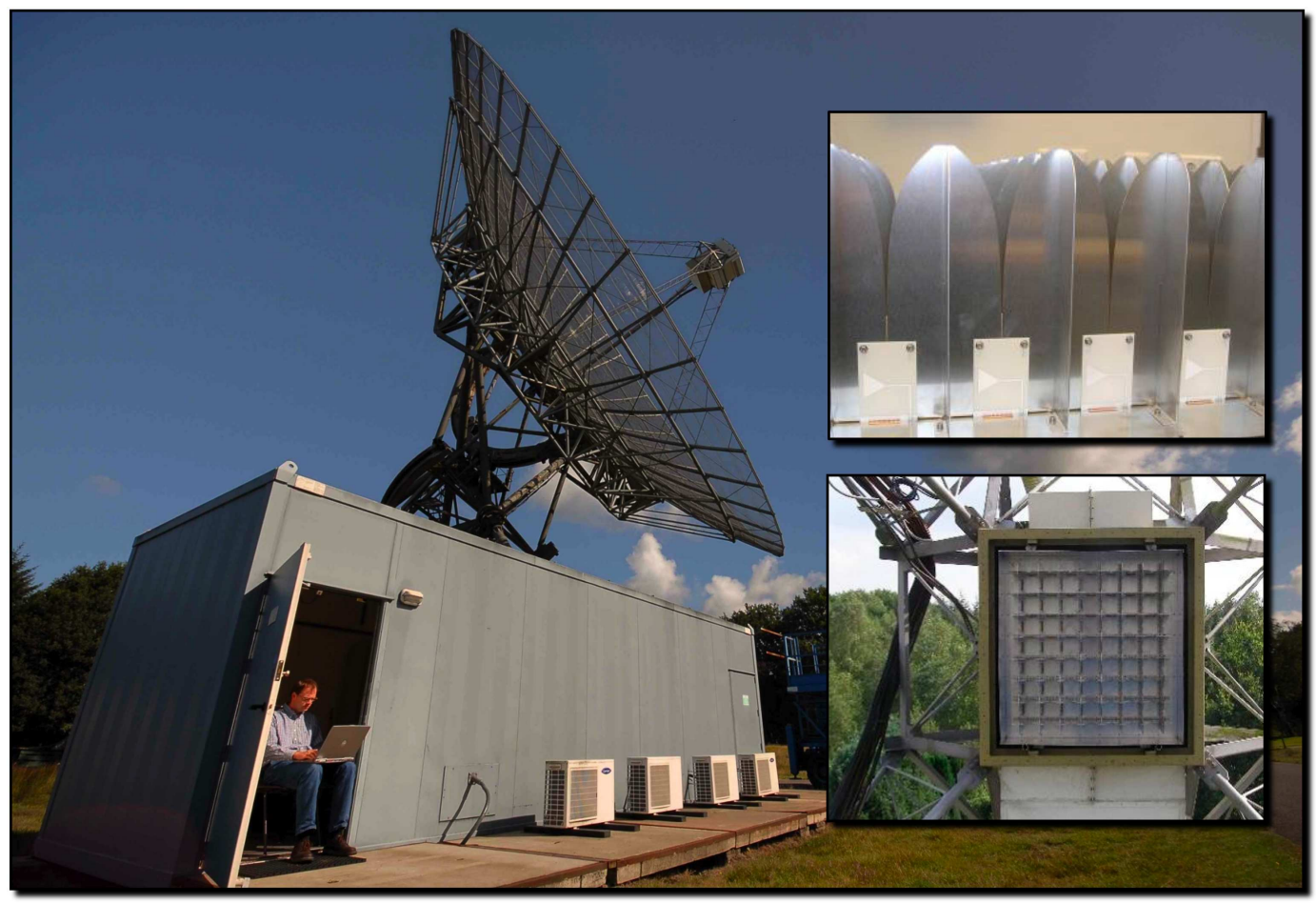

Figure 1: The WSRT telescope RT5 equipped with the APERTIF prototype. Shown in the inserts is the Vivaldi array in the lab (top right) and in the focal plane of the telescope (bottom right).

Australia Telescope National Facility. This opens the prospect of truly all-sky surveys with uniform properties in line and continuum. Apertif will become operational in 2012.

In this paper, we present results obtained with a prototype FPA. A prototype FPA, consisting of a dual-polarised Vivaldi array of $8 \times 7 \times 2$ elements, has been installed in the focal plane of one of the WSRT 25-m dishes. Each Vivaldi element has its own LNA. Due to the large physical size of the front-end system, these LNAs cannot be cryogenically cooled, leading to a higher system temperature compared to the current WSRT receivers. This, however, is largely offset by the higher aperture efficiency that FPAs offer. The system temperature of the current prototype is about 125 $\mathrm{K}$. In the fall of 2009 , on the path to the final design, a new prototype with much better performance $\left(T_{\text {sys }} \sim 70 \mathrm{~K}\right)$ will be installed. The data from the FPA elements are, after down-conversion, recorded and stored on disk. Much of the back-end of the prototype system consists of recycled hard- and software that earlier was used in the Lofar Initial Test System. The fact that the realtime signals are stored means that all further experiments, such as beamforming, can be done later off-line. This means that the same observation can be used for several independent technical experiments. A close up of this prototype in the focal plane of one of the WSRT telescopes is shown in Figure 1 (bottom right insert).

Figure 2 shows the beam patterns that were measured on the sky, in a single polarisation, for all elements of the FPA. Each panel covers the same $3^{\circ} \times 3^{\circ}$ on the sky and shows the primary beam of a given element. It is clear that each element is sensitive to a slightly different area on the sky and that together they cover about a region of $9 \mathrm{deg}^{2}$. One can clearly see the large optical distortions in the reception patterns of the elements that are close to the edge of the FPA. However, 


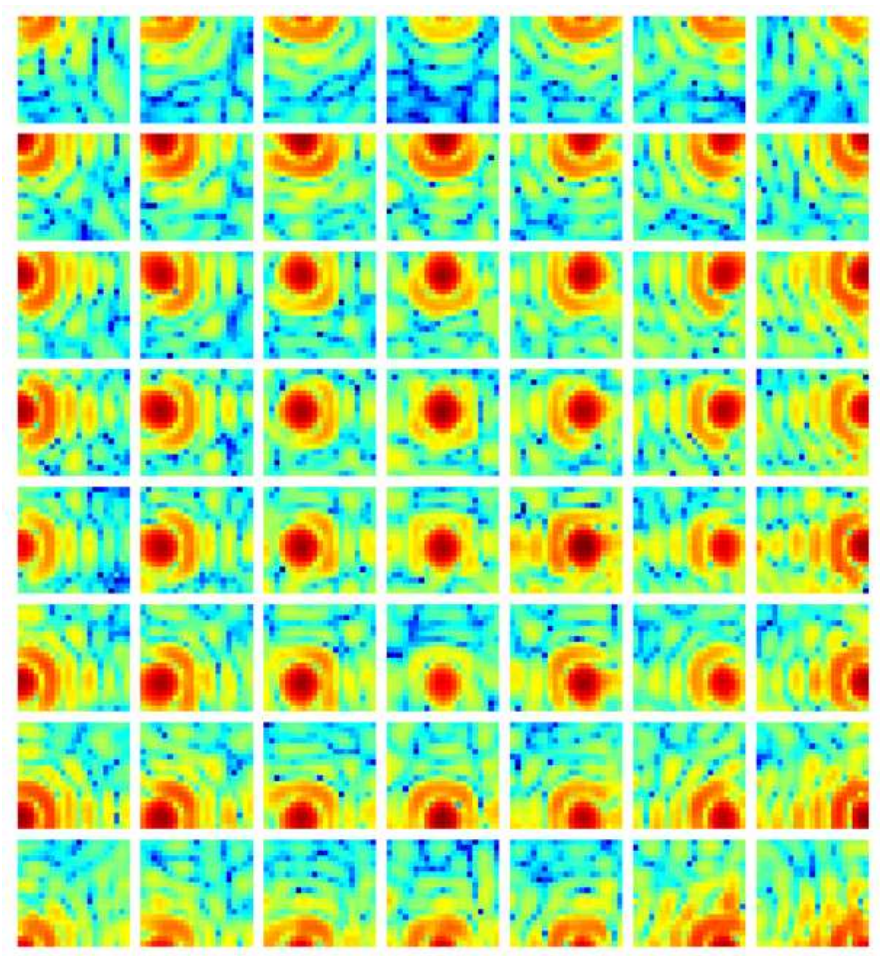

Figure 2: Response patterns on the sky of the individual Vivaldi elements of the FPA. Each panel covers the same $3^{\circ} \times 3^{\circ}$ on the sky and shows the primary beam of a given element.
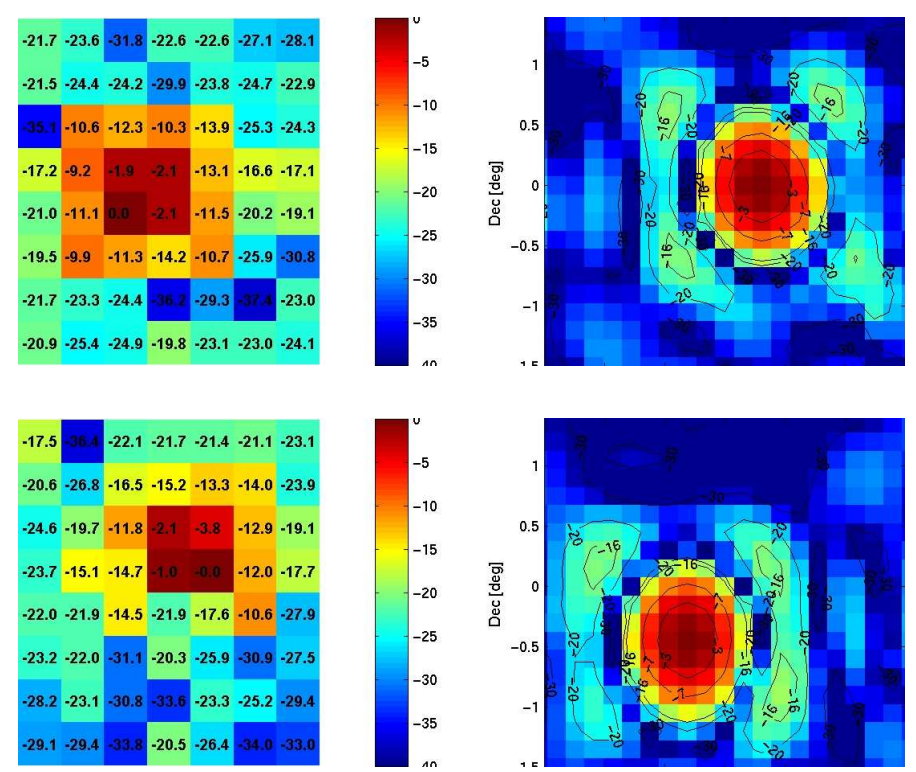

Figure 3: Optimised beam patterns (right) and weighting schemes (left) for two directions on the sky. Note the huge improvement in beam shape compared to those shown in Figure 2 


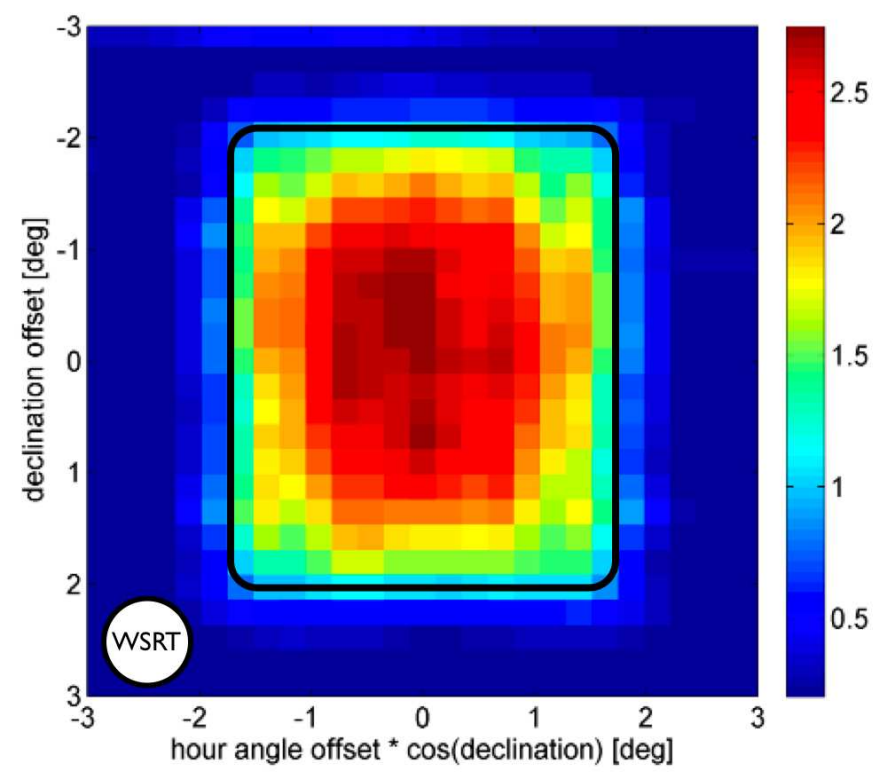

Figure 4: Sensitivity on the sky of the WSRT dish fitted with an FPA. The black line denotes the half-power points. For comparison, the field of view of the "old" WSRT system is also shown.

because the FPA fully samples the radiation field in the focal plane, the signals from all elements can be combined to optimise the response of the telescope into a certain direction. In this way, the aperture efficiency of the telescope can be much increased and the shape of the combined beam can be controlled. Therefore, even for telescopes where one wants to have only one beam on the sky, FPAs are a very effective technology. Moreover, this optimisation can be done for many directions simultaneously so that many optimised beams can be formed to cover a large region on the sky. This is the real power of radio telescopes fitted with FPAs. Examples are given in Figure 3. This figure shows the optimised beams for two directions, as well as the weights given to each element in order to form these optimised beam. Note that in this figure the same colour scale is used as in Figure 2. This shows that the sidelobe level of the optimised beams is much lower than that of the response of an individual element. Figure 3 also shows that the shapes of the optimised beams are much better than those of the elements themselves.

Figure 4 shows the sensitivity $(A / T)$ on the sky of the WSRT dish equipped with the prototype FPA. The black line shows the location of the half-power points and shows that the field of view is larger than $8 \mathrm{deg}^{2}$. For comparison, the field of view of the current WSRT is also shown. The huge gain in survey speed offered by the FPA system is obvious.

\section{First 'Light'}

First astronomical single dish observations with the Apertif prototype were performed on a number of sources. An excellent demonstration of the power of the FPA system is shown in Figure 5. The right panel shows a 163 pointing mosaic of M 31 obtained by Braun using the existing full WSRT with a single feed [2]. The left panel shows a single pointing with the APERTIF prototype on a single telescope. A total of $11 \times 11$ optimised beams were formed with the Vivaldi signals of 


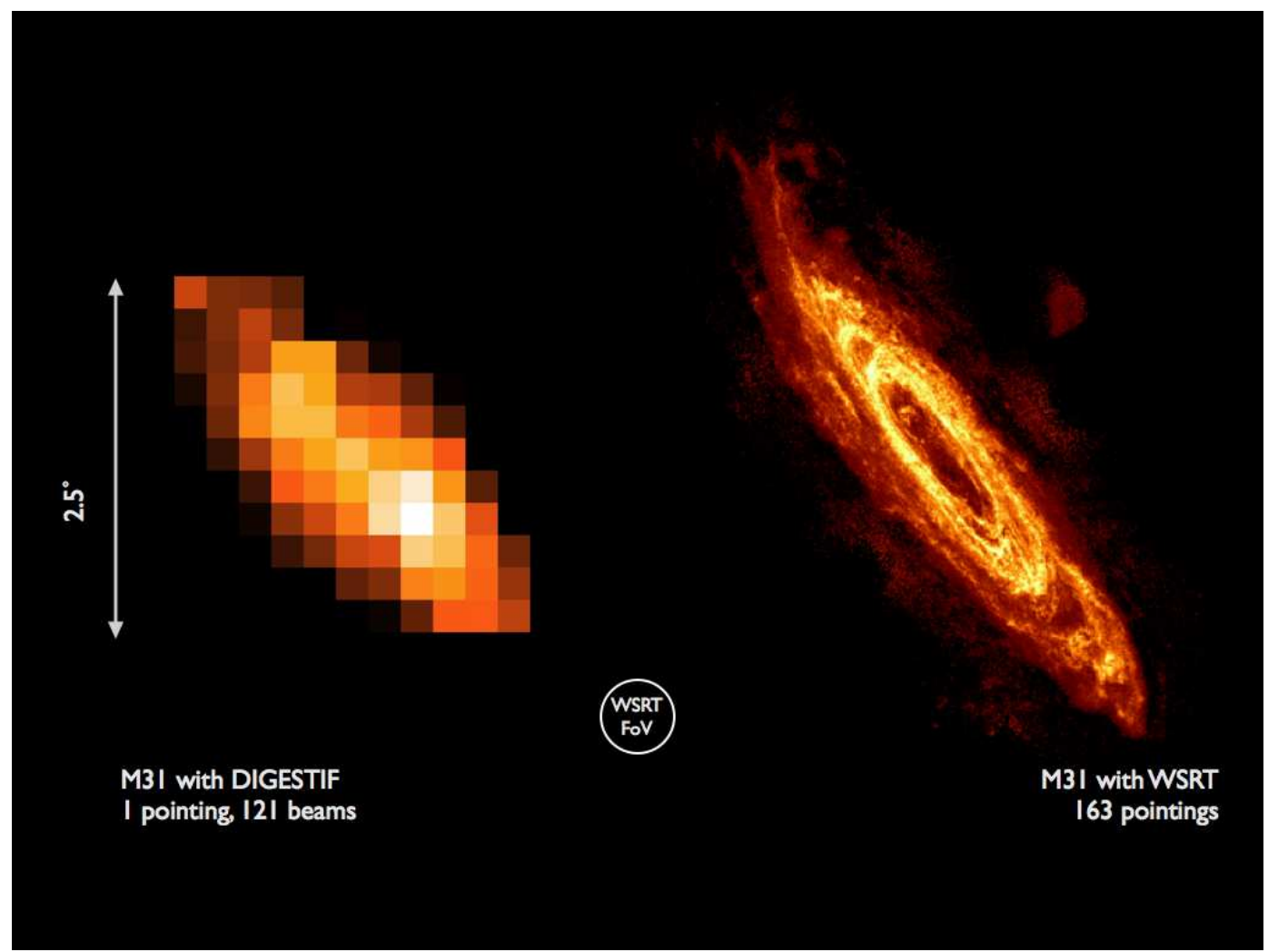

Figure 5: M 31 observed with the WSRT. Left: A single-pointing measurement with the Apertif prototype, yielding the resolution of a single dish but covering the area in one observation. Right: A 163-pointing observation in interferometric mode, yielding the full resolution of the 3-km array. Once all WSRT antennas are equipped with an FPA, the same high resolution can be achieved over the full field of view of Apertif.

a single telescope pointing. The implication is that when all WSRT telescopes are equipped with a FPA system, the 163 pointing observation can be carried out in only one or a few pointings, demonstrating the impressive increase in survey speed.

Further progress has been made by connecting the FPA equipped WSRT dish with 3 other WSRT dishes that still have the standard single-feed multi-frequency WSRT receiver (MFFE), and to use this to do interferometry. Figure 6 shows the fringes on 3C286 obtained by correlating the signal from an optimised FPA beam with that of a MFFE-equipped WSRT dish, as well as those between a single FPA element and an MFFE WSRT dish. For comparison, the fringes between two classic WSRT dishes is also shown. One can see that the amplitude of the fringes between optimised beam and the MFFE dish is about twice as large compared to those from the single FPA element correlated with the MFFE dish. This shows the effect of the beam optimisation. The difference in amplitude with the purely MFFE correlation is mainly due to the high system temperature of the FPA current prototype. This will improve when the new FPA prototype is installed.

Finally, we show an image that was made doing full interferometry over 12 hours using correlations between the FPA prototype and three MFFE equipped WSRT dishes. The image shows the two sources 3C 343 and 3C 343.1 that are separated by about 20 arcminutes on the sky. As far as we 

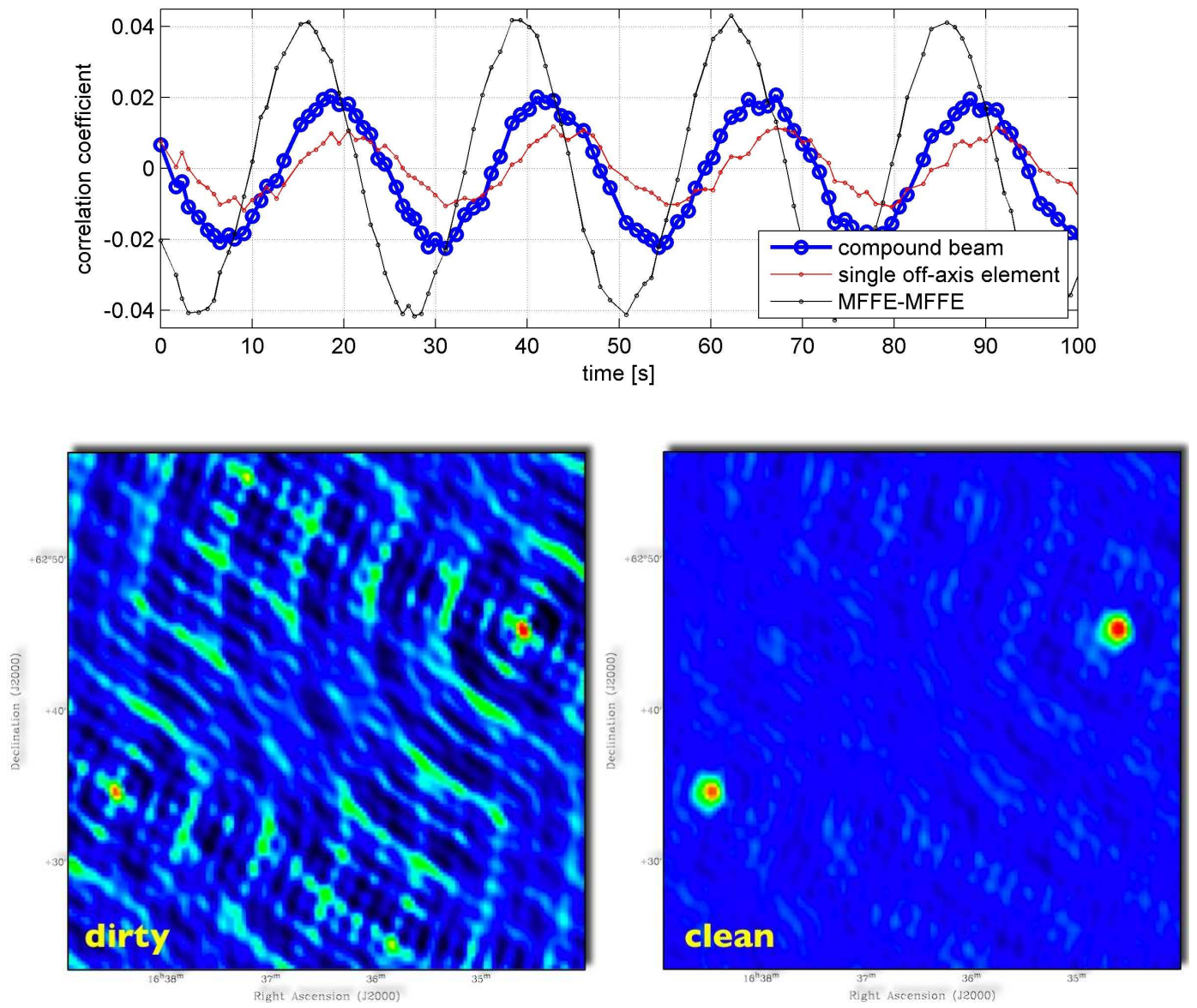

Figure 6: top: Fringes of 3C286 between an optimised beam of the FPA prototype and a MFFE WSRT dish, between a single FPA and the MFFE dish and between two MFFE equipped dishes. bottom: First interferometric image made using a radio dish with an FPA. To make this image, the signal of the optimised beam of the FPA prototype was correlated with 3 WSRT dishes that used the single-feed MFFE.

know, this is the first interferometric image ever made using a radio dish fitted with an FPA.

\section{Conclusions}

We have shown the results obtained with a prototype FPA installed on one of the WSRT dishes. We have demonstrated that the field of view can be enlarged with a large factor $(>30)$, we have also demonstrated that the high aperture efficiencies (75\%) can be achieved with FPAs while the results also indicate that the system temperature of the final system will be close to $50 \mathrm{~K}$. All this holds great promise for the final Apertif system which will turn the WSRT into a very effective survey instrument.

\section{References}

[1] J. G. Bij de Vaate, G. W. Kant, W. A. van Cappellen, S. van der Tol, "First Celestial Measurement Results of the Thousand Element Arra", URSI GA, Maastricht, August 2002 
[2] Braun, R., Thilker, D.A., Walterbos, R.A.M., Corbelli, E. 2009, ApJ, 295, 937

[3] Giovanelli, R., et al. 2005, AJ, 130, 2598

[4] Kant, G.W., Patel, P.D., Houwelingen, J.A. van, Ardenne, A. van, "Electronic Multi-Beam Radio Astronomy Concept: Embrace The European Demonstrator for the SKA Program", Proceedings URSI-GA 2005, New Delhi, India, www.ursi.org, October 23-29, 2005.

[5] Staveley-Smith, L., et al. 1996, Publications of the Astronomical Society of Australia, 13, 243

[6] Staveley-Smith, L., Wilson, W. E., Bird, T. S., Sinclair, M. W., Ekers, R. D., \& Webster, R. L. 1995, Multi-Feed Systems for Radio Telescopes, 75, 136

[7] Verheijen, M.A.W., Oosterloo, T.A., van Capellen, W., Heald, G., in Panoramic Radio Astronomy: Wide-field 1-2 GHz research on galaxy evolution. PoS (PRA2009) 010 